\title{
Risk factors for nephropathy in persons with type 1 diabetes: a population-based study
}

\author{
Shilan Seyed Ahmadi ${ }^{1,2,11}$ (D) Aldina Pivodic ${ }^{3,4} \cdot$ Ann-Marie Svensson $^{5} \cdot$ Hans Wedel $^{6} \cdot$ Björn Rathsman $^{7}$. \\ Thomas Nyström ${ }^{8} \cdot$ Johnny Ludvigsson ${ }^{9} \cdot$ Marcus Lind $^{1,2,10}$
}

Received: 21 September 2021 / Accepted: 19 December 2021 / Published online: 24 February 2022

(c) The Author(s) 2022

\begin{abstract}
Aims Albuminuria is strongly associated with risk of renal dysfunction, cardiovascular disease and mortality. However, clinical guidelines diverge, and evidence is sparse on what risk factor levels regarding blood pressure, blood lipids and BMI are needed to prevent albuminuria in adolescents and young adults with type 1 diabetes.

Methods A total of 9347 children and adults with type 1 diabetes [mean age 15.3 years and mean diabetes duration 1.4 years at start of follow-up] from The Swedish National Diabetes Registry were followed from first registration until end of 2017. Levels for risk factors for a risk increase in nephropathy were evaluated, and the gradient of risk per 1 SD (standard deviation) was estimated to compare the impact of each risk factor.

Results During the follow-up period, 8610 (92.1\%) remained normoalbuminuric, 737 (7.9\%) individuals developed micro- or macroalbuminuria at any time period of whom 132 (17.9\% of 737) individuals developed macroalbuminuria. Blood pressure $\geq 140 / 80 \mathrm{mmHg}$ was associated with increased risk of albuminuria $(p \leq 0.0001)$, as were triglycerides $\geq 1.0 \mathrm{mmol} / \mathrm{L}$ $(p=0.039)$, total cholesterol $\geq 5.0 \mathrm{mmol} / \mathrm{L}(p=0.0003), \mathrm{HDL}<1.0 \mathrm{mmol} / \mathrm{L}(p=0.013), \mathrm{LDL} 3.5-<4.0 \mathrm{mmol} / \mathrm{L}(p=0.020)$, and $\mathrm{BMI} \geq 30 \mathrm{~kg} / \mathrm{m}^{2}(p=0.033)$. HbA1c was the strongest risk factor for any albuminuria estimated by the measure gradient of risk per $1 \mathrm{SD}$, followed by diastolic blood pressure, triglycerides, systolic blood pressure, cholesterol and LDL. In patients with $\mathrm{HbAlc}>65 \mathrm{mmol} / \mathrm{mol}(>8.1 \%$ ), blood pressure $>140 / 70 \mathrm{mmHg}$ was associated with increased risk of albuminuria.

Conclusions Preventing renal complications in adolescents and young adults with type 1 diabetes need avoidance at relatively high levels of blood pressure, blood lipids and BMI, whereas very tight control is not associated with further risk reduction. For patients with long-term poor glycaemic control, stricter blood pressure control is advocated.
\end{abstract}

Keywords Type 1 diabetes $\cdot$ Albuminuria $\cdot$ Lipids $\cdot$ Blood pressure $\cdot$ BMI

\section{Introduction}

Diabetic nephropathy (DN), also known as diabetic kidney disease, occurs in $15-40 \%$ of all persons with type 1 diabetes. DN is characterised by pathological urinary albumin excretion, glomerular lesions, and loss of glomerular filtration rate [1]. Microalbuminuria has been established as an early marker of progressive kidney disease, and

This article belongs to the topical collection Diabetic Nephropathy, managed by Giuseppe Pugliese.

Johnny Ludvigsson, Marcus Lind shared last author position.

Shilan Seyed Ahmadi

shilan.seyed.ahmadi@vgregion.se

Extended author information available on the last page of the article macroalbuminuria an essential risk factor for cardiovascular disease, including stroke, atrial fibrillation, heart failure and mortality [2-4].

The Diabetes Control and Complication Trial (DCCT) and the follow-up Epidemiology of Diabetes Interventions and Complications (EDIC) demonstrated benefits of reducing $\mathrm{HbA} 1 \mathrm{c}$ and decreasing the risk of DN with intensive therapy compared with conventional therapy $[5,6]$. Blood pressure, dyslipidaemia, diabetes duration and albumin excretion rate have also been shown to be associated with progression of DN [7-16]. Recommendations regarding blood pressure diverge in guidelines from European Society of Cardiology (ESC), American College of Cardiology (ACC)/American Heart Association (AHA) and American Diabetes Association (ADA) [17-19]. Additionally, for blood lipid managements, evidence remains incomplete 
regarding adults with type 1 diabetes $<40$ years old, as earlier studies have mainly been performed in older patients with long diabetes duration [9-14]. To fully understand the impact of a risk factor for development of diabetes complications, it is essential to have information from diagnosis of diabetes and onwards in large, unselected population-based patient cohorts. Correct estimations of risk factors are essential for prognosis, but also for resource allocation and avoiding adverse events from unnecessary medications.

Recently, we found in a large patient cohort, following patients from diagnosis and onwards, that risks of nephropathy gradually increased with $\mathrm{HbA} 1 \mathrm{c}$ higher than $52 \mathrm{mmol} /$ mol $(7.0 \%)$ and no further risk reductions were seen at $\mathrm{HbA} 1 \mathrm{c}<48 \mathrm{mmol} / \mathrm{mol}(6.5 \%)$ [20]. In this population-based cohort study using paediatric and adult registries in Sweden, we aimed to evaluate what levels of blood lipids, blood pressure and BMI in persons with type 1 diabetes are related to risk of nephropathy. We also aimed to rank $\mathrm{HbAlc}$, blood lipids, blood pressure, body mass index (BMI), and smoking regarding their risk contribution to development of $\mathrm{DN}$.

\section{Methods}

We conducted a registry-based observational cohort study. Ethical approval was obtained from the Regional Ethical Review Board in Gothenburg, Sweden.

\section{Data sources}

Data were obtained from the paediatric registry, SWEDIABKIDS, and the adult registry, The Swedish National Diabetes Registry (NDR), which started in the years 2000 and 1996, respectively. The registries recently merged and include information on risk factors and complications. For inclusion in the registries, patients and/or guardians provide their informed consent. More than $95 \%$ of Swedish children and adolescents with type 1 diabetes are registered in the SWEDIABKIDS, the registries have included data on almost all children and adolescents with diabetes in Sweden [21]. In this study, information from NDR from 1998 onwards and for SWEDIABKIDS from 2000 onwards were included.

\section{Study population}

Current evaluations were performed from a recently used study cohort [20]. A total of 9347 persons with type 1 diabetes were included and followed up during period 1 January 1998 to 31 December 2017. Type 1 diabetes was defined as treatment with insulin and diagnosis $<30$ years of age, and this definition has been validated in $97 \%$ of cases [22]. To be included, children should have a clinical diagnosis of type 1 diabetes in the register. HLA and diabetes-related autoantibodies are determined in all children with newly diagnosed diabetes. In brief, criteria for inclusion in the study were children and adults who had their type 1 diabetes- diagnosis for $<5$ years when first recorded in the registries. In addition, a minimum of 4 visits with non-missing values during a minimum of 8-year follow-up from diagnosis were required.

\section{Study procedures}

Variables assessed were HbA1c (glycated haemoglobin), BMI (body mass index), low-density lipoprotein (LDL), high-density lipoprotein (HDL), cholesterol, triglycerides, systolic blood pressure (SBP), diastolic blood pressure (DBP) and smoking.

As earlier described [20], participants were included in the registers at different time points, whereby subcohorts were categorized by follow-up time from diabetes diagnosis to evaluate risk factors for a certain diabetes duration: 8-9, $10-11,12-13,14-15$, and 16-20 years. This approach was used to standardize the diabetes duration which is essential for the development of complications. Further, conventional approaches of evaluating time to event by time-updated models could not be used since hazard ratios were not constant over time (assumption of proportional hazards was not fulfilled). We followed participants from the first observation until the end of each subcohort if having a registration regarding albuminuria (normo-, micro- or macroalbuminuria). The primary analysis was a pooled analysis using all the five different subcohorts described above. Two separate analyses were performed for evaluating risk factors for any albuminuria (micro- or macroalbuminuria) and macroalbuminuria, respectively.

Microalbuminuria was defined as two positive tests from three samples taken within 1 year, with an albumin/creatinine ratio of 3-30 $\mathrm{mg} / \mathrm{mmol}(30-300 \mathrm{mg} / \mathrm{g})$ or U-albumin of $20-200 \mu \mathrm{g} / \mathrm{min}(20-300 \mathrm{mg} / \mathrm{L})$. Macroalbuminuria was defined as albumin/creatinine ratio $>30 \mathrm{mg} / \mathrm{mmol}$. If the first estimation of elevated albumin/creatinine ratio was registered as macroalbuminuria, this event was included in both the microalbuminuria and macroalbuminuria analyses.

According to instructions to the clinics, the registered blood pressure (SBP and DBP) was the mean value of two supine readings with a cuff of appropriate size and after at least $5 \mathrm{~min}$ of rest. $\mathrm{HbAlc}$ values were measured in $\mathrm{mmol} /$ mol and were also converted to levels in $\%$ according to the National Glycohaemoglobin Standardization Program for dual reporting [23]. Laboratory methods at participating care units for analysing HbA1c level and albuminuria are regularly checked with central reference samples of $\mathrm{HbA} 1 \mathrm{c}$ and albuminuria to ensure high accuracy [24].

SBP, DBP, LDL, HDL, triglycerides, total cholesterol, $\mathrm{BMI}$ and $\mathrm{HbA} 1 \mathrm{c}$ were evaluated as mean of longitudinally collected values, as continuous variables in relation to any 
albuminuria and to macroalbuminuria. Nonlinear effects were studied by analysing categories of the continuous variables; SBP categories $(<110 \mathrm{mmHg}, 110-<120 \mathrm{mmHg}$, $120-<130 \mathrm{mmHg}, 130-<140 \mathrm{mmHg}, \geq 140 \mathrm{mmHg}$ ), DBP categories $(<60 \mathrm{mmHg}, 60-<70 \mathrm{mmHg}, 70-<80 \mathrm{mmHg}$, $80-<85 \mathrm{mmHg}, \geq 85 \mathrm{mmHg})$, BMI categories $\left(<18.5 \mathrm{~kg} / \mathrm{m}^{2}\right.$, $18.5-<25 \mathrm{~kg} / \mathrm{m}^{2}, 25-<30 \mathrm{~kg} / \mathrm{m}^{2}, 30-<35 \mathrm{~kg} / \mathrm{m}^{2}, \geq 35 \mathrm{~kg} /$ $\left.\mathrm{m}^{2}\right)$, LDL categories $(<2.0 \mathrm{mmol} / \mathrm{l}, 2.0-<2.5 \mathrm{mmol} / \mathrm{L}$, $2.5-<3.0 \mathrm{mmol} / \mathrm{L}, 3.0-<3.5 \mathrm{mmol} / \mathrm{L}, 3.5-<4.0 \mathrm{~mm}$ $\mathrm{ol} / \mathrm{L}, \geq 4.0 \mathrm{mmol} / \mathrm{L})$, HDL categories $(<1.0 \mathrm{mmol} / \mathrm{L}$, $1.0-<1.5 \mathrm{mmol} / \mathrm{L}, 1.5-<2.0 \mathrm{mmol} / \mathrm{L}, \geq 2.0 \mathrm{mmol} / \mathrm{L})$, triglycerides categories $(<0.5 \mathrm{mmol} / \mathrm{L}, 0.5-<1.0 \mathrm{mmol} / \mathrm{L}$, $1.0-<1.5 \mathrm{mmol} / \mathrm{L}, 1.5-2.0 \mathrm{mmol} / \mathrm{L}, \geq 2.0 \mathrm{mmol} / \mathrm{L})$, cholesterol categories $(<4.0 \mathrm{mmol} / \mathrm{L}, 4.0-<5.0 \mathrm{mmol} / \mathrm{L}, 5.0-<6$. $0 \mathrm{mmol} / \mathrm{L}, \geq 6.0 \mathrm{mmol} / \mathrm{L}$ ) and smoking (no versus yes). Odds ratios were described for the following changes in the risk factors: SBP $(10 \mathrm{mmHg})$, DBP $(5 \mathrm{mmHg})$, blood lipid levels ( $1 \mathrm{mmol} / \mathrm{L})$, BMI $\left(5 \mathrm{~kg} / \mathrm{m}^{2}\right)$ and $\mathrm{HbA} 1 \mathrm{c}(10 \mathrm{mmol} / \mathrm{mol}, 1 \%)$. To rank the relative contribution of the risk factors, the metric gradient of risk per one SD was used [25] determining how much the risk of any and macroalbuminuria changes per one SD change in the risk factor. Additionally, sensitivity analyses on all persons $>13$ years of age at diabetes diagnosis were performed for the above-mentioned analyses. The impact of risk factor categories on any albuminuria and macroalbuminuria were further analysed after subgrouping for a mean level of $\mathrm{HbAlc}$ above or below $65 \mathrm{mmol} / \mathrm{mol}(8.1 \%)$ during follow-up.

\section{Statistical analysis}

Numbers and percentages (with 95\% confidence intervals) of complications were expressed for each category of SBP, DBP, BMI, LDL, HDL, triglycerides and cholesterol. Generalised estimating equations modelling was used to estimate the relation between the risk factors and endpoints for diabetic nephropathy, adjusting for within patient correlation for repeated data over the 5 follow-up cohorts. This method allows more than one observation per patient. Unstructured covariance matrix was used. The use of binomial distribution with logit link function resulted in odds ratios $(95 \%$ confidence intervals) as risk estimates. Analyses were performed for continuous variables and by categories of $\mathrm{HbAlc}, \mathrm{SBP}$, DBP, BMI, LDL, HDL, triglycerides and cholesterol, and for smoking. Risk factors were analysed adjusted for age and sex and additionally for mean HbAlc. Mean HbAlc was analysed adjusted for age and sex and additionally for SBP, BMI, triglycerides, cholesterol and smoking.

Pearson correlation was calculated when analysing association between two continuous variables.

No data were imputed. Tests were two-tailed and conducted at 0.05 significance level. All statistical programming was performed using SAS Software version 9.4 (SAS Institute, Cary, NC).

\section{Results}

\section{Patient characteristics}

A total of 9347 persons with type 1 diabetes were included. Patient characteristics are shown in Table 1 for the total cohort and for patients without albuminuria during followup and with micro- and/or macroalbuminuria at any time period during follow-up, respectively.

The proportion of women in the cohort was $43.9 \%$, the mean $\mathrm{HbAlc}$ for the longest follow-up was $63.2 \pm 11.4 \mathrm{mmol} / \mathrm{mol}(7.9 \pm 1.0 \%)$, mean blood pressure $116 \pm 9 / 70 \pm 6 \mathrm{mmHg}$, mean BMI $22.9 \pm 4.0 \mathrm{~kg} / \mathrm{m}^{2}$, mean LDL $2.5 \pm 0.7 \mathrm{mmol} / \mathrm{L}$, mean HDL $1.5 \pm 0.4 \mathrm{mmol} / \mathrm{L}$, mean cholesterol $4.5 \pm 0.8 \mathrm{mmol} / \mathrm{L}$, and mean triglycerides $1.1 \pm 0.7 \mathrm{mmol} / \mathrm{L}$. The mean age at first registered visit was $15.3 \pm 7.9$ years, and the mean duration of diabetes at first registration was $1.4 \pm 1.7$ years. Median follow-up was 12.0 years (range 8.0-20.0). In Supplemental Table 1, patient characteristics for the different subcohorts are shown.

\section{Relative contribution of risk factors for DN}

Among 9347 children and adults with type 1 diabetes from the Swedish National Diabetes Registry and observed over a median of 12.0 years, 737 (7.9\%) developed any albuminuria; 132 out of these 737 (17.9\%) developed macroalbuminuria. Levels of risk factors for a significant risk increase in albuminuria were evaluated and ranking of risk factors was estimated (Table 2). For both any albuminuria and macroalbuminuria, $\mathrm{HbA} 1 \mathrm{c}$ was the strongest risk factor. For any albuminuria, the remaining risk factors were ranked as follows: DBP, triglycerides, SBP, total cholesterol and LDL. No impact for: HDL, BMI and smoking. For macroalbuminuria, the remaining risk factors were ranked as follows: total cholesterol, SBP, DBP, and triglycerides. No impact existed for: BMI, LDL, HDL and smoking.

\section{Thresholds for risk of DN}

The variables were analysed as categorical variables for the impact on any albuminuria endpoints evaluated for different thresholds adjusted for age, sex and mean $\mathrm{HbAlc}$, as a study of potential nonlinear effects.

As seen in Fig. 1, the risk of any albuminuria increased at $\mathrm{SBP} \geq 140 \mathrm{mmHg}$ compared with the reference category $(110-120 \mathrm{mmHg}$ ) and at $\mathrm{DBP} \geq 80 \mathrm{mmHg}$ compared with the reference category $(60-<70 \mathrm{mmHg})$. For macroalbuminuria (Fig. 2), blood pressure $\geq 140 / 85 \mathrm{mmHg}$ 
Table 1 Patient characteristics

\begin{tabular}{|c|c|c|c|c|c|}
\hline Variable & All patients $(n=9347)$ & $\begin{array}{l}\text { All patients with } \\
\text { no albuminuria } \\
(n=8610)\end{array}$ & $\begin{array}{l}\text { All patients with } \\
\text { micro- or macroalbu- } \\
\text { minuria in any time } \\
\text { period }(n=737)\end{array}$ & $\begin{array}{l}\text { All patients with no } \\
\text { macroalbuminuria } \\
(n=9215)\end{array}$ & $\begin{array}{l}\text { All patients with } \\
\text { macroalbuminuria } \\
\text { in any time period } \\
(n=132)\end{array}$ \\
\hline \multicolumn{6}{|l|}{ Sex } \\
\hline Male & $5244(56.1 \%)$ & $4883(56.7 \%)$ & $361(49.0 \%)$ & $5184(56.3 \%)$ & $60(45.5 \%)$ \\
\hline Female & $4103(43.9 \%)$ & $3727(43.3 \%)$ & $376(51.0 \%)$ & $4031(43.7 \%)$ & $72(54.5 \%)$ \\
\hline Age at first visit & $15.3(7.9) n=9347$ & $15.2(7.9) n=8610$ & 16.0 (7.9) $n=737$ & $15.2(7.9) n=9215$ & $17.2(8.4) n=132$ \\
\hline Diabetes onset year & 2003 (4) $n=9347$ & 2003 (4) $n=8610$ & 2002 (4) $n=737$ & $2003(4) n=9215$ & $2002(4) n=132$ \\
\hline $\begin{array}{l}\text { HbA1c mean }(\%) \text { for } \\
\text { longest follow-up }\end{array}$ & $7.94(1.04) n=9347$ & $7.89(1.01) n=8610$ & 8.49 (1.27) $n=737$ & $7.93(1.03) n=9215$ & $8.57(1.40) n=132$ \\
\hline $\begin{array}{l}\mathrm{HbA1c} \text { mean }(\mathrm{mmol} / \\
\text { mol) for longest } \\
\text { follow-up }\end{array}$ & $63.2(11.4) n=9347$ & $62.7(11.0) n=8610$ & $69.3(13.9) n=737$ & $63.1(11.3) n=9215$ & $70.1(15.3) n=132$ \\
\hline \multicolumn{6}{|c|}{ HbAlc mean category for longest follow-up } \\
\hline$<48 \mathrm{mmol} / \mathrm{mol}$ & $667(7.1 \%)$ & $642(7.5 \%)$ & $25(3.4 \%)$ & $662(7.2 \%)$ & $5(3.8 \%)$ \\
\hline $48-52 \mathrm{mmol} / \mathrm{mol}$ & $932(10.0 \%)$ & $889(10.3 \%)$ & $43(5.8 \%)$ & $924(10.0 \%)$ & $8(6.1 \%)$ \\
\hline $53-57 \mathrm{mmol} / \mathrm{mol}$ & $1511(16.2 \%)$ & $1419(16.5 \%)$ & $92(12.5 \%)$ & $1498(16.3 \%)$ & $13(9.8 \%)$ \\
\hline $58-70 \mathrm{mmol} / \mathrm{mol}$ & $3963(42.4 \%)$ & $3701(43.0 \%)$ & $262(35.5 \%)$ & $3914(42.5 \%)$ & $49(37.1 \%)$ \\
\hline$>70 \mathrm{mmol} / \mathrm{mol}$ & $2274(24.3 \%)$ & $1959(22.8 \%)$ & $315(42.7 \%)$ & $2217(24.1 \%)$ & $57(43.2 \%)$ \\
\hline $\begin{array}{l}\mathrm{SBP} \text { mean }(\mathrm{mmHg}) \text { for } \\
\text { longest follow-up }\end{array}$ & 116.7 (8.8) $n=9330$ & $116.5(8.6) n=8594$ & $118.2(10.0) n=736$ & $116.6(8.7) n=9198$ & $119.1(12.0) n=132$ \\
\hline \multicolumn{6}{|c|}{ SBP mean category for longest follow-up } \\
\hline$<110 \mathrm{mmHg}$ & $2048(22.0 \%)$ & $1911(22.2 \%)$ & $137(18.6 \%)$ & $2021(22.0 \%)$ & $27(20.5 \%)$ \\
\hline $110-<120 \mathrm{mmHg}$ & $4129(44.3 \%)$ & $3812(44.4 \%)$ & $317(43.1 \%)$ & $4074(44.3 \%)$ & $55(41.7 \%)$ \\
\hline $120-<130 \mathrm{mmHg}$ & $2490(26.7 \%)$ & $2286(26.6 \%)$ & $204(27.7 \%)$ & $2461(26.8 \%)$ & $29(22.0 \%)$ \\
\hline $130-<140 \mathrm{mmHg}$ & $567(6.1 \%)$ & $512(6.0 \%)$ & $55(7.5 \%)$ & $556(6.0 \%)$ & $11(8.3 \%)$ \\
\hline$>=140 \mathrm{mmHg}$ & $96(1.0 \%)$ & $73(0.8 \%)$ & $23(3.1 \%)$ & $86(0.9 \%)$ & $10(7.6 \%)$ \\
\hline $\begin{array}{l}\text { DBP mean }(\mathrm{mmHg}) \\
\text { for longest follow-up }\end{array}$ & $70.1(5.9) n=9330$ & $69.9(5.8) n=8594$ & $71.7(6.8) n=736$ & $70.0(5.8) n=9198$ & $72.4(8.2) n=132$ \\
\hline \multicolumn{6}{|c|}{ DBP mean category for longest follow-up } \\
\hline$<60 \mathrm{mmHg}$ & $317(3.4 \%)$ & $298(3.5 \%)$ & $19(2.6 \%)$ & $314(3.4 \%)$ & $3(2.3 \%)$ \\
\hline $60-<70 \mathrm{mmHg}$ & $4425(47.4 \%)$ & $4133(48.1 \%)$ & $292(39.7 \%)$ & $4368(47.5 \%)$ & $57(43.2 \%)$ \\
\hline $70-<80 \mathrm{mmHg}$ & $4086(43.8 \%)$ & $3741(43.5 \%)$ & $345(46.9 \%)$ & $4036(43.9 \%)$ & $50(37.9 \%)$ \\
\hline $80-<85 \mathrm{mmHg}$ & $397(4.3 \%)$ & $347(4.0 \%)$ & $50(6.8 \%)$ & $388(4.2 \%)$ & $9(6.8 \%)$ \\
\hline$>=85 \mathrm{mmHg}$ & $105(1.1 \%)$ & $75(0.9 \%)$ & $30(4.1 \%)$ & $92(1.0 \%)$ & $13(9.8 \%)$ \\
\hline $\begin{array}{l}\text { BMI mean }(\mathrm{kg} / \mathrm{m} 2) \text { for } \\
\text { longest follow-up }\end{array}$ & $22.9(4.0) n=9316$ & $22.9(4.0) n=8583$ & 23.6 (4.9) $n=733$ & $22.9(4.0) n=9185$ & $23.9(5.0) n=131$ \\
\hline \multicolumn{6}{|c|}{ BMI mean category for longest follow-up } \\
\hline$<18.5 \mathrm{~kg} / \mathrm{m}^{\wedge} 2$ & $1038(11.1 \%)$ & $961(11.2 \%)$ & $77(10.5 \%)$ & $1025(11.2 \%)$ & $13(9.9 \%)$ \\
\hline $18.5-<25 \mathrm{~kg} / \mathrm{m}^{\wedge} 2$ & $5880(63.1 \%)$ & $5450(63.5 \%)$ & $430(58.7 \%)$ & $5804(63.2 \%)$ & $76(58.0 \%)$ \\
\hline $25-<30 \mathrm{~kg} / \mathrm{m}^{\wedge} 2$ & $1917(20.6 \%)$ & $1762(20.5 \%)$ & $155(21.1 \%)$ & $1889(20.6 \%)$ & $28(21.4 \%)$ \\
\hline $30-<35 \mathrm{~kg} / \mathrm{m}^{\wedge} 2$ & $383(4.1 \%)$ & $336(3.9 \%)$ & $47(6.4 \%)$ & $373(4.1 \%)$ & $10(7.6 \%)$ \\
\hline$>=35 \mathrm{~kg} / \mathrm{m}^{\wedge} 2$ & $98(1.1 \%)$ & $74(0.9 \%)$ & $24(3.3 \%)$ & $94(1.0 \%)$ & $4(3.1 \%)$ \\
\hline $\begin{array}{l}\text { HDL mean (mmol/L) } \\
\text { for longest follow-up }\end{array}$ & $1.52(0.38) n=8534$ & $1.52(0.38) n=7844$ & $1.47(0.38) n=690$ & $1.52(0.38) n=8413$ & $1.48(0.42) n=121$ \\
\hline \multicolumn{6}{|c|}{ HDL mean category for longest follow-up } \\
\hline$<1.0 \mathrm{mmol} / \mathrm{L}$ & $383(4.5 \%)$ & $332(4.2 \%)$ & $51(7.4 \%)$ & $372(4.4 \%)$ & $11(9.1 \%)$ \\
\hline $1.0-<1.5 \mathrm{mmol} / \mathrm{L}$ & $4026(47.2 \%)$ & $3681(46.9 \%)$ & $345(50.0 \%)$ & $3971(47.2 \%)$ & $55(45.5 \%)$ \\
\hline $1.5-<2.0 \mathrm{mmol} / \mathrm{L}$ & $3233(37.9 \%)$ & $2998(38.2 \%)$ & $235(34.1 \%)$ & $3191(37.9 \%)$ & $42(34.7 \%)$ \\
\hline$>=2.0 \mathrm{mmol} / \mathrm{L}$ & $892(10.5 \%)$ & $833(10.6 \%)$ & $59(8.6 \%)$ & $879(10.4 \%)$ & $13(10.7 \%)$ \\
\hline $\begin{array}{l}\text { LDL mean }(\mathrm{mmol} / \mathrm{L}) \\
\text { for longest follow-up }\end{array}$ & $2.53(0.69) n=8539$ & $2.51(0.68) n=7846$ & $2.65(0.73) n=693$ & $2.52(0.68) n=8422$ & $2.72(0.79) n=117$ \\
\hline
\end{tabular}


Table 1 (continued)

\begin{tabular}{|c|c|c|c|c|c|}
\hline Variable & All patients $(n=9347)$ & $\begin{array}{l}\text { All patients with } \\
\text { no albuminuria } \\
(n=8610)\end{array}$ & $\begin{array}{l}\text { All patients with } \\
\text { micro- or macroalbu- } \\
\text { minuria in any time } \\
\text { period }(n=737)\end{array}$ & $\begin{array}{l}\text { All patients with no } \\
\text { macroalbuminuria } \\
(n=9215)\end{array}$ & $\begin{array}{l}\text { All patients with } \\
\text { macroalbuminuria } \\
\text { in any time period } \\
(n=132)\end{array}$ \\
\hline \multicolumn{6}{|c|}{ LDL mean category for longest follow-up } \\
\hline$<2.0 \mathrm{mmol} / \mathrm{L}$ & $1832(21.5 \%)$ & $1709(21.8 \%)$ & $123(17.7 \%)$ & $1812(21.5 \%)$ & $20(17.1 \%)$ \\
\hline $2.0-<2.5 \mathrm{mmol} / \mathrm{L}$ & $2617(30.6 \%)$ & $2427(30.9 \%)$ & $190(27.4 \%)$ & $2589(30.7 \%)$ & $28(23.9 \%)$ \\
\hline $2.5-<3.0 \mathrm{mmol} / \mathrm{L}$ & $2191(25.7 \%)$ & $2013(25.7 \%)$ & $178(25.7 \%)$ & $2159(25.6 \%)$ & $32(27.4 \%)$ \\
\hline $3.0-<3.5 \mathrm{mmol} / \mathrm{L}$ & $1175(13.8 \%)$ & $1061(13.5 \%)$ & $114(16.5 \%)$ & $1155(13.7 \%)$ & $20(17.1 \%)$ \\
\hline $3.5-<4.0 \mathrm{mmol} / \mathrm{L}$ & $501(5.9 \%)$ & $440(5.6 \%)$ & $61(8.8 \%)$ & $490(5.8 \%)$ & $11(9.4 \%)$ \\
\hline$>=4.0 \mathrm{mmol} / \mathrm{L}$ & $223(2.6 \%)$ & $196(2.5 \%)$ & $27(3.9 \%)$ & $217(2.6 \%)$ & $6(5.1 \%)$ \\
\hline $\begin{array}{l}\text { Cholesterol mean } \\
\text { (mmol/L) for longest } \\
\text { follow-up }\end{array}$ & $4.49(0.78) n=8707$ & $4.48(0.77) n=8010$ & $4.68(0.85) n=697$ & $4.49(0.78) n=8587$ & $4.89(0.99) n=120$ \\
\hline \multicolumn{6}{|c|}{ Cholesterol mean category for longest follow-up } \\
\hline$<4.0 \mathrm{mmol} / \mathrm{L}$ & $2250(25.8 \%)$ & $2107(26.3 \%)$ & $143(20.5 \%)$ & $2231(26.0 \%)$ & $19(15.8 \%)$ \\
\hline $4.0-<5.0 \mathrm{mmol} / \mathrm{L}$ & $4394(50.5 \%)$ & $4074(50.9 \%)$ & $320(45.9 \%)$ & $4344(50.6 \%)$ & $50(41.7 \%)$ \\
\hline $5.0-<6.0 \mathrm{mmol} / \mathrm{L}$ & $1721(19.8 \%)$ & $1533(19.1 \%)$ & $188(27.0 \%)$ & $1684(19.6 \%)$ & $37(30.8 \%)$ \\
\hline$>=6.0 \mathrm{mmol} / \mathrm{L}$ & $342(3.9 \%)$ & $296(3.7 \%)$ & $46(6.6 \%)$ & $328(3.8 \%)$ & $14(11.7 \%)$ \\
\hline $\begin{array}{l}\text { Triglycerides mean } \\
\text { (mmol/L) for longest } \\
\text { follow-up }\end{array}$ & $1.07(0.68) n=8300$ & $1.04(0.59) n=7630$ & $1.38(1.24) n=670$ & $1.06(0.64) n=8189$ & $1.75(1.94) n=111$ \\
\hline \multicolumn{6}{|c|}{ Triglycerides mean category for longest follow-up } \\
\hline$<0.5 \mathrm{mmol} / \mathrm{L}$ & $335(4.0 \%)$ & $317(4.2 \%)$ & $18(2.7 \%)$ & $333(4.1 \%)$ & $2(1.8 \%)$ \\
\hline $0.5-<1.0 \mathrm{mmol} / \mathrm{L}$ & $4550(54.8 \%)$ & $4267(55.9 \%)$ & $283(42.2 \%)$ & $4513(55.1 \%)$ & $37(33.3 \%)$ \\
\hline $1.0-<1.5 \mathrm{mmol} / \mathrm{L}$ & $2175(26.2 \%)$ & $1980(26.0 \%)$ & $195(29.1 \%)$ & $2148(26.2 \%)$ & $27(24.3 \%)$ \\
\hline $1.5-2.0 \mathrm{mmol} / \mathrm{L}$ & $687(8.3 \%)$ & $613(8.0 \%)$ & $74(11.0 \%)$ & $670(8.2 \%)$ & $17(15.3 \%)$ \\
\hline$>=2.0 \mathrm{mmol} / \mathrm{L}$ & $553(6.7 \%)$ & $453(5.9 \%)$ & $100(14.9 \%)$ & $525(6.4 \%)$ & $28(25.2 \%)$ \\
\hline \multicolumn{6}{|c|}{ Smoking at any time before for longest follow-up } \\
\hline No & $6518(77.8 \%)$ & $6021(78.4 \%)$ & $497(71.3 \%)$ & $6436(78.0 \%)$ & $82(65.1 \%)$ \\
\hline Yes & $1862(22.2 \%)$ & $1662(21.6 \%)$ & $200(28.7 \%)$ & $1818(22.0 \%)$ & $44(34.9 \%)$ \\
\hline
\end{tabular}

For categorical variables $n(\%)$ is presented.

For continuous variables Mean (SD) $/ n=$ is presented.

increased the risk. For blood lipids, the risk of any albuminuria increased for LDL $3.5-<4.0 \mathrm{mmol} / \mathrm{L}$, $\mathrm{HDL}<1.0 \mathrm{mmol} / \mathrm{L}$, triglycerides $1.0-<1.5 \mathrm{mmol} / \mathrm{L}$, and cholesterol $5.0-<6.0 \mathrm{mmol} / \mathrm{L}$ compared to each reference category. For BMI, the risk of any albuminuria increased at $\geq 30 \mathrm{~kg} / \mathrm{m}^{2}$. For macroalbuminuria, mean triglycerides and cholesterol remained significant risk factors for the highest category compared to the respective reference category. Associations between BMI categories and macroalbuminuria, showed numerically higher OR, although not being significant.

In the supplementary appendix, the categorized levels of SBP, DBP, LDL, HDL, triglycerides, cholesterol and BMI analysed for the impact on albuminuria endpoints are shown and, additionally, adjusted for age and sex (Supplemental Table 2).

\section{Impact of risk factors on subgrouping of $\mathrm{HbA1c}$}

The impact of various risk factors on albuminuria was also evaluated based on mean $\mathrm{HbA} 1 \mathrm{c}$ levels during follow-up ( $\leq 65 \mathrm{mmol} / \mathrm{mol}$ and $>65 \mathrm{mmol} / \mathrm{mol}[8.1 \%]$ ) adjusted for age, sex and mean HbAlc, as shown in Supplemental Table 3. For persons with mean $\mathrm{HbAlc}>65 \mathrm{mmol} / \mathrm{mol}$ during follow-up, a blood pressure $>140 / 70 \mathrm{mmHg}$ was associated with increased risk of albuminuria, whereas for persons with $\mathrm{HbA} 1 \mathrm{c} \leq 65 \mathrm{mmol} / \mathrm{mol}$, blood pressure $>140 / 80 \mathrm{mmHg}$ was associated with increased risk.

\section{Sensitivity analysis}

Similar patterns were observed in a sensitivity analysis when patients $>13$ years of age at diabetes diagnosis were included 
Table 2 Generalized estimating equation (GEE) models for the impact of risk factors on nephropathy endpoints

\begin{tabular}{|c|c|c|c|c|c|c|c|c|}
\hline \multirow[b]{2}{*}{ Variable } & \multirow[b]{2}{*}{$\begin{array}{l}n(\%)(95 \% \mathrm{CI}) \\
\text { events** }\end{array}$} & \multirow[b]{2}{*}{$\begin{array}{l}n(\%)(95 \% \mathrm{CI}) \\
\text { persons with } \\
\text { events*** }\end{array}$} & \multicolumn{3}{|c|}{ Adjusted for age and sex } & \multicolumn{3}{|c|}{ Multivariable adjusted* } \\
\hline & & & $\begin{array}{l}\text { OR }(95 \% \mathrm{CI}) \\
\text { per specified } \\
\text { unit increase }\end{array}$ & $\begin{array}{l}\text { OR }(95 \% \\
\text { CI) per } 1 \mathrm{SD} \\
\text { increase }\end{array}$ & $p$-value & $\begin{array}{l}\text { OR }(95 \% \mathrm{CI}) \\
\text { per specified } \\
\text { unit increase }\end{array}$ & $\begin{array}{l}\text { OR }(95 \% \\
\text { CI }) \text { per } 1 \mathrm{SD} \\
\text { increase }\end{array}$ & $p$-value \\
\hline \multicolumn{9}{|c|}{ Microalbuminuria/Macroalbuminuria vs None } \\
\hline $\begin{array}{l}\text { Mean HbA1c } \\
\text { (mmol/mol) } \\
\text { (by } 10 \text { unit } \\
\text { increase) }\end{array}$ & $\begin{array}{l}1027(6.1 \%) \\
(5.7 \%-6.5 \%)\end{array}$ & $\begin{array}{l}737(7.9 \%) \\
\quad(7.3 \%-8.4 \%)\end{array}$ & $\begin{array}{l}1.55(1.46- \\
1.66)\end{array}$ & $\begin{array}{l}1.65(1.53- \\
1.78)\end{array}$ & $<.0001$ & $\begin{array}{l}1.49(1.39- \\
1.61)\end{array}$ & $\begin{array}{l}1.58(1.45- \\
1.72)\end{array}$ & $<.0001$ \\
\hline $\begin{array}{l}\text { Mean HbA1c } \\
\text { (\%) (by } 1 \text { unit } \\
\text { increase) }\end{array}$ & $\begin{array}{l}1027(6.1 \%) \\
\quad(5.7 \%-6.5 \%)\end{array}$ & $\begin{array}{l}737(7.9 \%) \\
\quad(7.3 \%-8.4 \%)\end{array}$ & $\begin{array}{l}1.62(1.51- \\
1.73)\end{array}$ & $\begin{array}{l}1.65(1.53- \\
1.77)\end{array}$ & $<.0001$ & $\begin{array}{l}1.55(1.43- \\
1.68)\end{array}$ & $\begin{array}{l}1.58(1.45- \\
1.71)\end{array}$ & $<.0001$ \\
\hline $\begin{array}{l}\text { Mean SBP } \\
\text { (mmHg) } \\
\text { (by } 10 \text { unit } \\
\text { increase) }\end{array}$ & $\begin{array}{l}1026(6.1 \%) \\
(5.7 \%-6.5 \%)\end{array}$ & $\begin{array}{l}736(7.9 \%) \\
\quad(7.3 \%-8.5 \%)\end{array}$ & $\begin{array}{l}1.28(1.16- \\
1.42)\end{array}$ & $\begin{array}{l}1.24(1.13- \\
1.35)\end{array}$ & $<.0001$ & $\begin{array}{l}1.25(1.12- \\
1.38)\end{array}$ & $\begin{array}{l}1.21(1.10- \\
1.32)\end{array}$ & $<.0001$ \\
\hline $\begin{array}{l}\text { Mean DBP } \\
\text { (mmHg) (by } 5 \\
\text { unit increase) }\end{array}$ & $\begin{array}{l}1026(6.1 \%) \\
(5.7 \%-6.5 \%)\end{array}$ & $\begin{array}{l}736(7.9 \%) \\
\quad(7.3 \%-8.5 \%)\end{array}$ & $\begin{array}{l}1.30(1.21- \\
1.41)\end{array}$ & $\begin{array}{l}1.35(1.24- \\
1.48)\end{array}$ & $<.0001$ & $\begin{array}{l}1.23(1.14- \\
1.34)\end{array}$ & $\begin{array}{l}1.27(1.16- \\
1.39)\end{array}$ & $<.0001$ \\
\hline $\begin{array}{l}\text { Mean BMI (kg/ } \\
\text { m2) (by } 5 \text { unit } \\
\text { increase) }\end{array}$ & $\begin{array}{l}1022(6.1 \%) \\
(5.7 \%-6.5 \%)\end{array}$ & $\begin{array}{l}733(7.9 \%) \\
\quad(7.3 \%-8.4 \%)\end{array}$ & $\begin{array}{l}1.19(1.06- \\
1.34)\end{array}$ & $\begin{array}{l}1.15(1.05- \\
1.25)\end{array}$ & 0.0031 & $\begin{array}{l}1.10(0.98- \\
1.23)\end{array}$ & $\begin{array}{l}1.07(0.98- \\
1.17)\end{array}$ & 0.12 \\
\hline $\begin{array}{c}\text { Mean LDL } \\
\text { (mmol/L) } \\
\text { (by } 1 \text { unit } \\
\text { increase) }\end{array}$ & $\begin{array}{l}957(6.2 \%) \\
\quad(5.8 \%-6.5 \%)\end{array}$ & $\begin{array}{l}689(8.1 \%) \\
\quad(7.5 \%-8.7 \%)\end{array}$ & $\begin{array}{l}1.32(1.18- \\
1.47)\end{array}$ & $\begin{array}{l}1.21(1.12- \\
1.31)\end{array}$ & $<.0001$ & $\begin{array}{l}1.17(1.05- \\
1.30)\end{array}$ & $\begin{array}{l}1.11(1.03- \\
1.20)\end{array}$ & 0.0056 \\
\hline $\begin{array}{c}\text { Mean HDL } \\
\text { (mmol/L) } \\
\text { (by } 1 \text { unit } \\
\text { increase) }\end{array}$ & $\begin{array}{l}959(6.2 \%) \\
\quad(5.8 \%-6.6 \%)\end{array}$ & $\begin{array}{l}688(8.1 \%) \\
\quad(7.5 \%-8.7 \%)\end{array}$ & $\begin{array}{l}0.68(0.51- \\
0.89)\end{array}$ & $\begin{array}{l}0.86(0.77- \\
0.95)\end{array}$ & 0.0046 & $\begin{array}{l}0.82(0.63- \\
1.05)\end{array}$ & $\begin{array}{l}0.92(0.84- \\
1.02)\end{array}$ & 0.12 \\
\hline $\begin{array}{l}\text { Mean triglycer- } \\
\text { ides (mmol/L) } \\
\text { (by } 1 \text { unit } \\
\text { increase) }\end{array}$ & $\begin{array}{l}930(6.2 \%) \\
\quad(5.8 \%-6.6 \%)\end{array}$ & $\begin{array}{l}668(8.0 \%) \\
\quad(7.5 \%-8.7 \%)\end{array}$ & $\begin{array}{l}1.58(1.44- \\
1.73)\end{array}$ & $\begin{array}{l}1.35(1.27- \\
1.44)\end{array}$ & $<.0001$ & $\begin{array}{l}1.37(1.25- \\
1.50)\end{array}$ & $\begin{array}{l}1.23(1.16- \\
1.31)\end{array}$ & $<.0001$ \\
\hline $\begin{array}{l}\text { Mean choles- } \\
\text { terol }(\mathrm{mmol} / \mathrm{L}) \\
\text { (by } 1 \text { unit } \\
\text { increase) }\end{array}$ & $\begin{array}{l}968(6.1 \%) \\
\quad(5.8 \%-6.5 \%)\end{array}$ & $\begin{array}{l}695(8.0 \%) \\
\quad(7.4 \%-8.6 \%)\end{array}$ & $\begin{array}{l}1.36(1.24- \\
1.51)\end{array}$ & $\begin{array}{l}1.28(1.19- \\
1.39)\end{array}$ & $<.0001$ & $\begin{array}{l}1.20(1.09- \\
1.32)\end{array}$ & $\begin{array}{l}1.16(1.07- \\
1.25)\end{array}$ & 0.0002 \\
\hline $\begin{array}{l}\text { Smoking at any } \\
\text { time before } \\
\text { (yes vs no) }\end{array}$ & $\begin{array}{l}975(6.2 \%) \\
\quad(5.9 \%-6.6 \%)\end{array}$ & $\begin{array}{l}695(8.3 \%) \\
\quad(7.7 \%-8.9 \%)\end{array}$ & $\begin{array}{l}1.42(1.19- \\
1.69)\end{array}$ & $\begin{array}{l}1.15(1.07- \\
1.24)\end{array}$ & $<.0001$ & $\begin{array}{l}1.05(0.88- \\
1.26)\end{array}$ & $\begin{array}{l}1.02(0.95- \\
1.10)\end{array}$ & 0.58 \\
\hline \multicolumn{9}{|c|}{ Macroalbuminuria vs None/Microalbuminuria } \\
\hline $\begin{array}{l}\text { Mean HbA1c } \\
\text { (mmol/mol) } \\
\text { (by } 10 \text { unit } \\
\text { increase) }\end{array}$ & $\begin{array}{l}163(1.0 \%) \\
\quad(0.8 \%-1.1 \%)\end{array}$ & $\begin{array}{l}132(1.4 \%) \\
\quad(1.2 \%-1.7 \%)\end{array}$ & $\begin{array}{l}1.59(1.35- \\
1.86)\end{array}$ & $\begin{array}{l}1.69(1.41- \\
2.03)\end{array}$ & $<.0001$ & $\begin{array}{l}1.46(1.22- \\
1.74)\end{array}$ & $\begin{array}{l}1.54(1.26- \\
1.88)\end{array}$ & $<.0001$ \\
\hline $\begin{array}{l}\text { Mean HbA1c } \\
\text { (\%) (by } 1 \text { unit } \\
\text { increase) }\end{array}$ & $\begin{array}{l}163(1.0 \%) \\
\quad(0.8 \%-1.1 \%)\end{array}$ & $\begin{array}{l}132(1.4 \%) \\
\quad(1.2 \%-1.7 \%)\end{array}$ & $\begin{array}{l}1.66(1.39- \\
1.98)\end{array}$ & $\begin{array}{l}1.69(1.41- \\
2.03)\end{array}$ & $<.0001$ & $\begin{array}{l}1.51(1.25- \\
1.83)\end{array}$ & $\begin{array}{l}1.54(1.26- \\
1.88)\end{array}$ & $<.0001$ \\
\hline $\begin{array}{l}\text { Mean SBP } \\
\text { (mmHg) } \\
\text { (by } 10 \text { unit } \\
\text { increase) }\end{array}$ & $\begin{array}{l}163(1.0 \%) \\
\quad(0.8 \%-1.1 \%)\end{array}$ & $\begin{array}{l}132(1.4 \%) \\
\quad(1.2 \%-1.7 \%)\end{array}$ & $\begin{array}{l}1.47(1.13- \\
1.90)\end{array}$ & $\begin{array}{l}1.39(1.11- \\
1.73)\end{array}$ & 0.0037 & $\begin{array}{l}1.40(1.09- \\
1.80)\end{array}$ & $\begin{array}{l}1.33(1.08- \\
1.65)\end{array}$ & 0.0083 \\
\hline $\begin{array}{l}\text { Mean DBP } \\
\text { (mmHg) (by } 5 \\
\text { unit increase) }\end{array}$ & $\begin{array}{l}163(1.0 \%) \\
\quad(0.8 \%-1.1 \%)\end{array}$ & $\begin{array}{l}132(1.4 \%) \\
\quad(1.2 \%-1.7 \%)\end{array}$ & $\begin{array}{l}1.36(1.12- \\
1.65)\end{array}$ & $\begin{array}{l}1.42(1.14- \\
1.76)\end{array}$ & 0.0017 & $\begin{array}{l}1.28(1.05- \\
1.56)\end{array}$ & $\begin{array}{l}1.32(1.06- \\
1.66)\end{array}$ & 0.014 \\
\hline
\end{tabular}


Table 2 (continued)

\begin{tabular}{|c|c|c|c|c|c|c|c|c|}
\hline \multirow[b]{2}{*}{ Variable } & \multirow[b]{2}{*}{$\begin{array}{l}n(\%)(95 \% \mathrm{CI}) \\
\text { events** }\end{array}$} & \multirow[b]{2}{*}{$\begin{array}{l}n(\%)(95 \% \mathrm{CI}) \\
\text { persons with } \\
\text { events*** }\end{array}$} & \multicolumn{3}{|c|}{ Adjusted for age and sex } & \multicolumn{3}{|c|}{ Multivariable adjusted* } \\
\hline & & & $\begin{array}{l}\text { OR }(95 \% \mathrm{CI}) \\
\text { per specified } \\
\text { unit increase }\end{array}$ & $\begin{array}{l}\text { OR }(95 \% \\
\text { CI) per } 1 \mathrm{SD} \\
\text { increase }\end{array}$ & $p$-value & $\begin{array}{l}\text { OR }(95 \% \mathrm{CI}) \\
\text { per specified } \\
\text { unit increase }\end{array}$ & $\begin{array}{l}\text { OR }(95 \% \\
\text { CI) per } 1 \mathrm{SD} \\
\text { increase }\end{array}$ & $p$-value \\
\hline $\begin{array}{l}\text { Mean BMI (kg/ } \\
\text { m2) (by } 5 \text { unit } \\
\text { increase) }\end{array}$ & $\begin{array}{l}162(1.0 \%) \\
(0.8 \%-1.1 \%)\end{array}$ & $\begin{array}{l}131(1.4 \%) \\
\quad(1.2 \%-1.7 \%)\end{array}$ & $\begin{array}{l}1.15(0.90- \\
1.47)\end{array}$ & $\begin{array}{l}1.11(0.92- \\
1.35)\end{array}$ & 0.27 & $\begin{array}{l}1.04(0.80- \\
1.35)\end{array}$ & $\begin{array}{l}1.03(0.84- \\
1.26)\end{array}$ & 0.78 \\
\hline $\begin{array}{c}\text { Mean LDL } \\
\text { (mmol/L) } \\
\text { (by } 1 \text { unit } \\
\text { increase) }\end{array}$ & $\begin{array}{l}144(0.9 \%) \\
\quad(0.8 \%-1.1 \%)\end{array}$ & $\begin{array}{l}116(1.4 \%) \\
\quad(1.1 \%-1.6 \%)\end{array}$ & $\begin{array}{l}1.38(1.03- \\
1.84)\end{array}$ & $\begin{array}{l}1.25(1.02- \\
1.53)\end{array}$ & 0.031 & $\begin{array}{l}1.27(0.98- \\
1.66)\end{array}$ & $\begin{array}{l}1.18(0.98- \\
1.42)\end{array}$ & 0.076 \\
\hline $\begin{array}{c}\text { Mean HDL } \\
\text { (mmol/L) } \\
\text { (by } 1 \text { unit } \\
\text { increase) }\end{array}$ & $\begin{array}{l}148(1.0 \%) \\
\quad(0.8 \%-1.1 \%)\end{array}$ & $\begin{array}{l}120(1.4 \%) \\
\quad(1.2 \%-1.7 \%)\end{array}$ & $\begin{array}{l}0.65(0.32- \\
1.31)\end{array}$ & $\begin{array}{l}0.84(0.64- \\
1.11)\end{array}$ & 0.23 & $\begin{array}{l}0.89(0.45- \\
1.74)\end{array}$ & $\begin{array}{l}0.96(0.73- \\
1.24)\end{array}$ & 0.73 \\
\hline $\begin{array}{l}\text { Mean triglycer- } \\
\text { ides (mmol/L) } \\
\text { (by } 1 \text { unit } \\
\text { increase) }\end{array}$ & $\begin{array}{l}137(0.9 \%) \\
\quad(0.8 \%-1.1 \%)\end{array}$ & $\begin{array}{l}110(1.3 \%) \\
\quad(1.1 \%-1.6 \%)\end{array}$ & $\begin{array}{l}1.66(1.44- \\
1.91)\end{array}$ & $\begin{array}{l}1.40(1.27- \\
1.53)\end{array}$ & $<.0001$ & $\begin{array}{l}1.50(1.31- \\
1.72)\end{array}$ & $\begin{array}{l}1.31(1.20- \\
1.43)\end{array}$ & $<.0001$ \\
\hline $\begin{array}{l}\text { Mean choles- } \\
\text { terol }(\mathrm{mmol} / \mathrm{L}) \\
\text { (by } 1 \text { unit } \\
\text { increase) }\end{array}$ & $\begin{array}{l}147(0.9 \%) \\
\quad(0.8 \%-1.1 \%)\end{array}$ & $\begin{array}{l}119(1.4 \%) \\
\quad(1.1 \%-1.6 \%)\end{array}$ & $\begin{array}{l}1.64(1.28- \\
2.09)\end{array}$ & $\begin{array}{l}1.48(1.22- \\
1.81)\end{array}$ & $<.0001$ & $\begin{array}{l}1.49(1.21- \\
1.84)\end{array}$ & $\begin{array}{l}1.38(1.16- \\
1.63)\end{array}$ & 0.0002 \\
\hline $\begin{array}{l}\text { Smoking at any } \\
\text { time before } \\
\text { (yes vs no) }\end{array}$ & $\begin{array}{l}156(1.0 \%) \\
\quad(0.8 \%-1.2 \%)\end{array}$ & $\begin{array}{l}125(1.5 \%) \\
\quad(1.2 \%-1.8 \%)\end{array}$ & $\begin{array}{l}1.81(1.23- \\
2.67)\end{array}$ & $\begin{array}{l}1.28(1.09- \\
1.50)\end{array}$ & 0.0027 & $\begin{array}{l}1.30(0.87- \\
1.95)\end{array}$ & $\begin{array}{l}1.11(0.94- \\
1.31)\end{array}$ & 0.21 \\
\hline
\end{tabular}

*HbA1c mean adjusted for age, sex, SBP, BMI, triglycerides, cholesterol and smoking. All other variables adjusted for age, sex and HbA1c mean.

**The number of events pooled over all periods.

***The number of individuals with at least one event pooled over all periods.

regarding their impact on development of any albuminuria. The patient characteristics are shown in the Supplemental Table 4. In brief, 4606 patients were included in the analyses. Numerically, they had a lower mean HbAlc, higher mean blood pressure, comparable mean blood lipids and higher mean BMI at baseline compared to the full cohort population. The same target levels for blood pressure $(\geq 140 / 80 \mathrm{mmHg})$ and BMI $\left(\geq 30 \mathrm{~kg} / \mathrm{m}^{2}\right)$ were associated with increased risk of any albuminuria (Supplement Table 5).

As observed in Supplemental Table 6, the impact of continuous risk factors expressed by per specified unit and 1 SD increase on nephropathy endpoints are shown. For any albuminuria, HbA1c followed by DBP, SBP, triglycerides, BMI and HDL remained the significant risk factors. For macroalbuminuria, blood pressure and triglycerides had a significant impact, but not other studied variables.

\section{Conclusions}

In this population-based cohort study, we used both pediatric and adult registries to evaluate the impact of various risk factors on the development of albuminuria in persons with type 1 diabetes. HbA1c was the strongest risk factor for any albuminuria, followed by blood pressure, blood lipids and BMI. No association was seen for smoking. For macroalbuminuria, systolic and diastolic blood pressure, triglycerides and cholesterol remained significant non-glycaemic risk factors for the development of albuminuria. In persons with poor glycaemic control (mean HbA1c $>65 \mathrm{mmol} / \mathrm{mol}$ [8.1\%]), blood pressure $>140 / 70 \mathrm{mmHg}$ was associated with increased risk of albuminuria.

In 1993, DCCT showed beneficial effects of reducing $\mathrm{HbA} 1 \mathrm{c}$ and decreasing the risk of nephropathy with intensive therapy compared with conventional therapy [5]. In the follow-up EDIC-study, the beneficial effects on albumin excretion and the reduced incidence of hypertension after the DCCT suggested that previous intensive treatment had extended benefit in delaying progression of diabetic nephropathy [6]. The importance of glycaemic control on nephropathy has been shown in several other studies [7-12]. Since then, multiple studies have evaluated other risk factors for progression of albuminuria. Hypertension, dyslipidaemia, and diabetes duration are well-known risk factors, whereas the impact of gender, smoking and BMI have shown diverging results [7-12]. Recently, a study, using the DCCT/ 


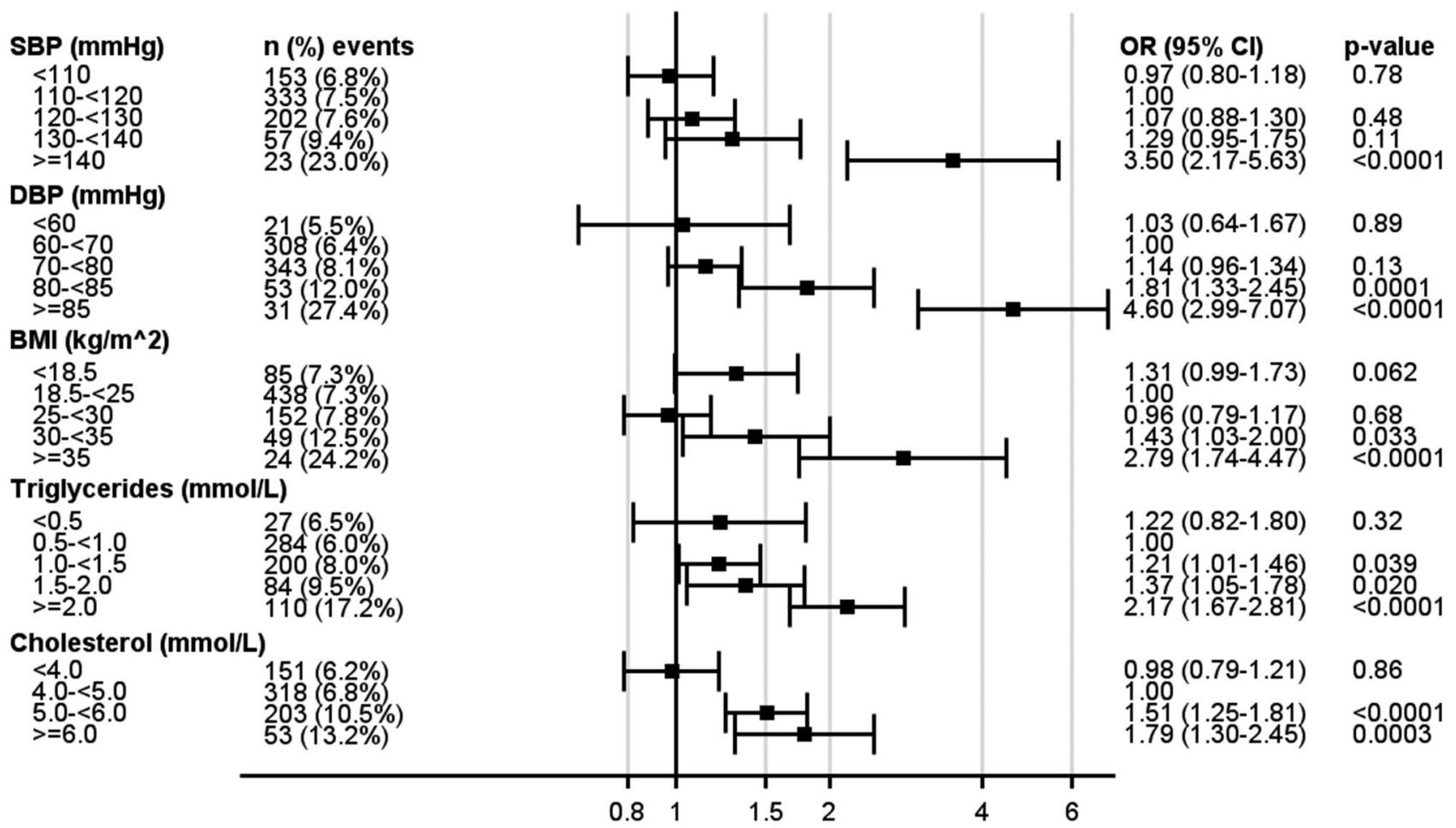

Fig. 1 Generalized Estimating Equation (GEE) models for the impact of various variable categories on any albuminuria

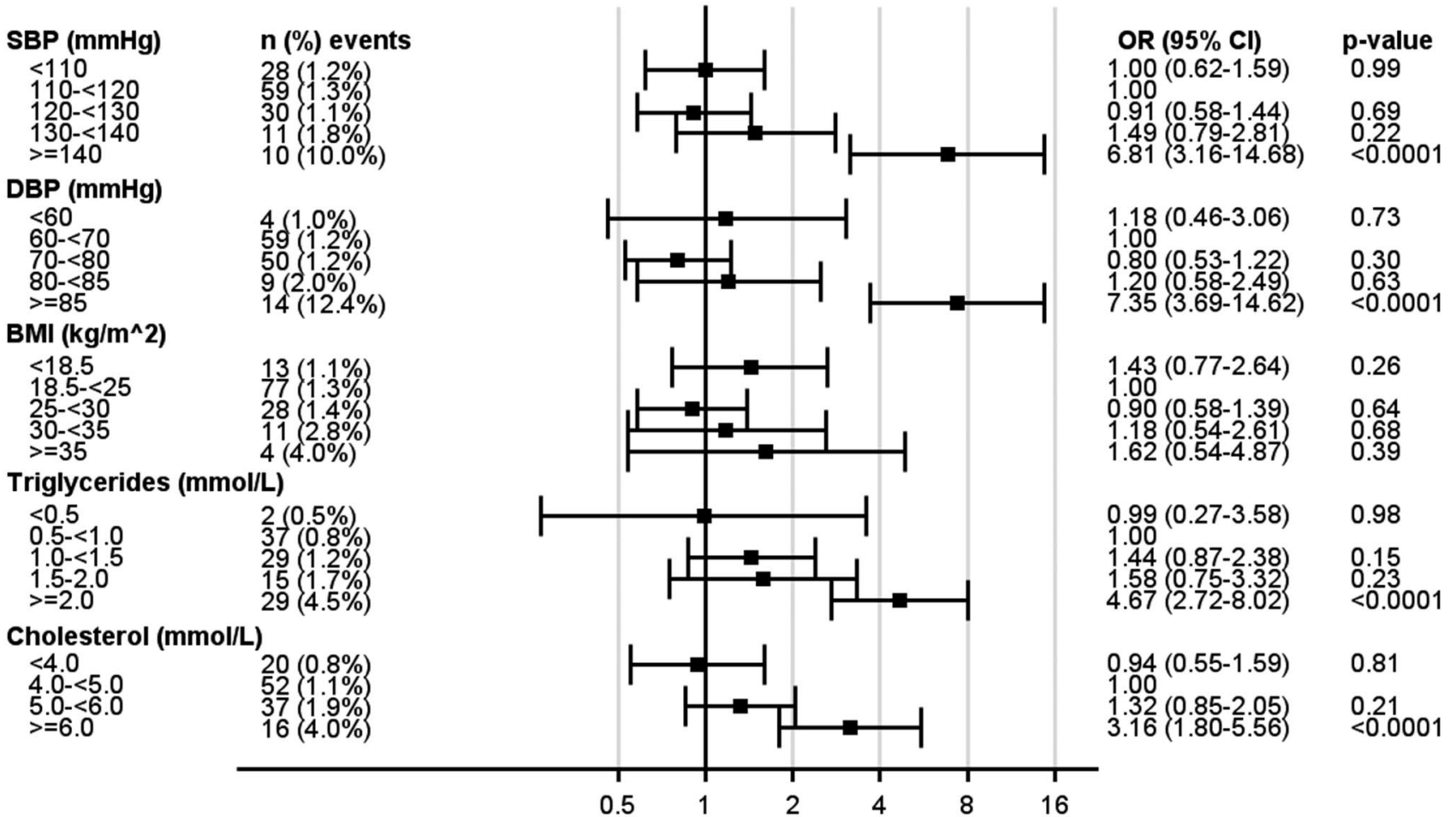

Fig. 2 Generalized Estimating Equation (GEE) models for the impact of various variable categories on macroalbuminuria 
EDIC cohort, showed that the risk of macroalbuminuria was associated with sex, blood pressure and lipids (LDL, HDL and triglycerides) [16]. Our results expand upon the results from prior studies by providing data from persons with type 1 diabetes in a population-based contemporary cohort with short diabetes duration at baseline (mean 1.4 years) followed over 8-20 years, as well as what levels of these risk factors increases the risk of albuminuria.

In our study, SBP and DBP were evaluated both as continuous and categorical variables. Both analyses showed that blood pressure has an important impact on the risk of albuminuria. As a continuous variable, an increase in $10 \mathrm{mmHg}$ for mean SBP increased the risk of any albuminuria by $25 \%$ and for mean DBP by $35 \%$ with an increase in each $5 \mathrm{mmHg}$. When evaluating SBP and DBP as a categorical variable, levels above 140/80 increased risk of albuminuria. For $\mathrm{DBP} \geq 85 \mathrm{mmHg}$, the OR was increased to 4.6. According to guidelines from ADA and ESC, hypertension is defined as $\geq 140 / 90 \mathrm{mmHg}$, whereas ACC/AHA define hypertension as $>130 / 90 \mathrm{mmHg}$. Additionally, the guidelines differ in recommendations regarding risk assessment and target goals in adults. Further, the recommendations are mainly based on clinical trials on an older population with or without type 2 diabetes [17-19]. Hence, few high-quality data exist for blood pressure targets in type 1 diabetes from diagnosis and onwards.

Another important risk factor for albuminuria was blood lipids. Our results show that the risk of albuminuria increased at following levels: triglycerides $\geq 1.0 \mathrm{mmol} / \mathrm{L}$, total cholesterol $\geq 5.0 \mathrm{mmol} / \mathrm{L}, \mathrm{LDL} 3.5-<4.0 \mathrm{mmol} / \mathrm{L}$ and $\mathrm{HDL}<1.0 \mathrm{mmol} / \mathrm{L}$ compared to each group selected reference. Of the blood lipids, triglycerides had the strongest impact on risk of albuminuria after $\mathrm{HbA} 1 \mathrm{c}$ and diastolic blood pressure. In a cross-sectional analysis, it was shown that glycaemic control was an important mediator of lipid abnormalities in youth with type 1 diabetes [26]. Additionally, in the FinnDiane study, it was shown that triglyceride and cholesterol levels were associated with incident albuminuria [27]. The mechanisms responsible for the dyslipidaemia in type 1 diabetes remain unclear, but the subcutaneous route of insulin administration, that is responsible for peripheral hyperinsulinemia, may play a role [28]. Another possible explanation is weight gain, and central obesity from a sedentary lifestyle. The individuals with type 1 diabetes and an associated metabolic syndrome have an increased cardiovascular risk compared to other type 1 diabetes patients related to the development of atherogenic lipid profile, in which hypertriglyceridemia is an essential component [29].

In clinical practice, caregivers are more hesitant to treat younger individuals with medications for increased blood pressure and blood lipids. There is a risk of side effects with medications, evidence is less robust due to lack of randomized trials in this patient group and younger individuals have lower adherence to treatment [30]. It is, therefore, important to know at what levels risk factors besides $\mathrm{HbA} 1 \mathrm{c}$ really increases the risk of albuminuria. Systolic blood pressure of 130 compared to $140 \mathrm{mmHg}$ has been debated for long periods [31]. This study supports the higher blood pressure level, but at repeated determinations above these values, younger individuals should also get antihypertensive drugs. Additionally, since risk factors can be more difficult to judge among young children, we analysed in a sensitivity analysis patient with diabetes diagnosis after 13 years of age, the same age span as in the DCCT study, with confirming results. In several diseases, strict risk factor targets have been found to be beneficial in prevention to more severe disease, e.g. lower blood lipid targets after myocardial infarction. In the current study, patients with high mean $\mathrm{HbA1c}$ ( $>8.1 \%$ ) during follow-up seem to benefit of an even lower diastolic blood pressure of $<70 \mathrm{mmHg}$. Hence, stricter blood pressure control is indicated in persons with type 1 diabetes with poor glycaemic control.

In the case of statins, these are more commonly used at slightly older ages when the risk of cardiovascular disease increases. With clearly increased LDL and cholesterol levels in younger individuals, one should first exclude familial hypercholesterolemia and then consider medication if dietary advice is not enough [32]. Regarding BMI, previous studies have not found that obesity is a strong risk factor for myocardial infarction and mortality [33]. However, the risk of heart failure, which is significantly more common in type 1 diabetes than in the general population, clearly increases above BMI 30 [34, 35]. In addition, in a study from National Diabetes Audit data, which included individuals with type 1 and type 2 diabetes in UK and Wales, association between obesity and kidney disease was shown [36]. The fact that we see that the risk of albuminuria increases above BMI 30 indicates that an important focus in diabetes care among adolescents and young adults should be to avoid obesity. Furthermore, a weight reduction can contribute to reduced blood pressure in obese patients [37].

A strength of the current study is that patients were followed from close after type 1 diabetes diagnosis (mean diabetes duration 1.4 years at inclusion) over 8-20 years. It is essential to have the complete historical risk factor profile from patients before development of diabetes complications. However, this study was limited by the absence of other factors contributing to albuminuria which were not documented in the registry (e.g., other renal diseases). Since the current study was not randomized, residual confounding cannot be excluded. However, since randomized trials are lacking in the current age group, this first large population-based study following non-glycaemic risk factors from diagnosis of type 1 diabetes is an important contribution to the evidence for risk factor targets in adolescents and young adults with type 1 diabetes. 
In this analysis of nationwide registers, it was shown that beside the importance of $\mathrm{HbAlc}$, prevention of renal complications in adolescents and young adults with type 1 diabetes was associated with certain target levels for nonglycaemic risk factors: blood pressure $\geq 140 / 80 \mathrm{mmHg}$, LDL $3.5-<4.0 \mathrm{mmol} / \mathrm{L}, \mathrm{BMI} \geq 30 \mathrm{~kg} / \mathrm{m}^{2}, \mathrm{HDL}<1.0 \mathrm{mmol} / \mathrm{L}$ and total cholesterol $\geq 5.0 \mathrm{mmol} / \mathrm{L}$. Risk was evident already at triglycerides levels $\geq 1.0 \mathrm{mmol} / \mathrm{L}$, which had the strongest impact on risk after glycaemic control and diastolic blood pressure. The current findings are essential for patients and care-givers where treatment for blood pressure, lipids and obesity have potential adverse effects and require major health care resources. Somewhat more tight blood pressure control is indicated in patients with poor glycaemic control being at high risk of nephropathy.

Supplementary Information The online version contains supplementary material available at https://doi.org/10.1007/s00592-022-01863-6.

Author contributions SSA wrote the first draft of the manuscript. AP performed statistical calculations. All authors were involved in the study design, interpretation of data, and review of the manuscript.

Funding Open access funding provided by University of Gothenburg. This study was supported by the Swedish government (Agreement for Medical Education and Research) and Novo Nordisk Foundation. The funders had no role in the study design, data collection, data analysis, data interpretation, or writing of the report.

Data availability Data are not publicly available. Data may be available on request to the Swedish National Diabetes Registry.

\section{Code availability N/A.}

\section{Declarations}

Conflict of interest TN has received honoraria on expert group participation from AstraZeneca, Merck Shark \& Dohme, Novo Nordisk, Eli Lilly and Company, Boerhinger Ingelheim, Abbot and Amgen. ML has received grants from Eli Lilly and Novo Nordisk, and consulting fees from AstraZeneca, Boehringer Ingelheim, Dexcom, Eli Lilly, MSD and Novo Nordisk. SSA, AP, AS, HW, BR, JL: no conflicts of interests.

Consent to participate Each patient provides informed consent for inclusion in the NDR

\section{Consent for publication Not required.}

Ethics approval This study was approved by the regional ethical and review board of the University of Gothenburg, Gothenburg, Sweden (Dnr 977-17).

Open Access This article is licensed under a Creative Commons Attribution 4.0 International License, which permits use, sharing, adaptation, distribution and reproduction in any medium or format, as long as you give appropriate credit to the original author(s) and the source, provide a link to the Creative Commons licence, and indicate if changes were made. The images or other third party material in this article are included in the article's Creative Commons licence, unless indicated otherwise in a credit line to the material. If material is not included in the article's Creative Commons licence and your intended use is not permitted by statutory regulation or exceeds the permitted use, you will need to obtain permission directly from the copyright holder. To view a copy of this licence, visit http://creativecommons.org/licenses/by/4.0/.

\section{References}

1. Lim A (2014) Diabetic nephropathy-Complications and treatment. Int J Nephrol Renov Dis 7:361-381. https://doi.org/10. 2147/IJNRD.S40172

2. de Boer IH, Gao X, Cleary PA et al (2016) Albuminuria changes and cardiovascular and renal outcomes in type 1 diabetes: the DCCT/EDIC Study. Clin J Am Soc Nephrol CJASN 11(11):19691977. https://doi.org/10.2215/CJN.02870316

3. Hallström S, Pivodic A, Rosengren A, Ólafsdóttir AF, Svensson AM, Lind M (2019) Risk factors for atrial fibrillation in people with type 1 diabetes: an observational cohort study of 36,258 patients from the swedish national diabetes registry. Diabetes Care 42(8):1530-1538. https://doi.org/10.2337/dc18-2457

4. Vestberg D, Rosengren A, Olsson M et al (2015) Decreased eGFR as a risk factor for heart failure in 13781 individuals with type 1 diabetes. J Diabetes Sci Technol 10(1):131-136. https://doi.org/ $10.1177 / 1932296815596174$

5. Nathan DM, Genuth S, Lachin J, Diabetes Control and Complications Trial Research Group et al (1993) The effect of intensive treatment of diabetes on the development and progression of longterm complications in insulin-dependent diabetes mellitus. New Engl J Med 329(14):977-986. https://doi.org/10.1056/NEJM1 99309303291401

6. DCCT/EDIC research group (2014) Effect of intensive diabetes treatment on albuminuria in type 1 diabetes: long-term follow-up of the diabetes control and complications trial and epidemiology of diabetes interventions and complications study. Lancet Diabetes Endocrinol 2(10):793-800. https://doi.org/10.1016/S22138587(14)70155-X

7. Coonrod BA, Ellis D, Becker DJ et al (1993) Predictors of microalbuminuria in individuals with IDDM. Pittsburgh epidemiology of diabetes complications study. Diabetes Care 16(10):1376-1383. https://doi.org/10.2337/diacare.16.10.1376

8. Bojestig M, Arnqvist HJ, Hermansson G, Karlberg BE, Ludvigsson J (1994) Declining incidence of nephropathy in insulindependent diabetes mellitus. N Engl J Med 330(1):15-18. https:// doi.org/10.1056/NEJM199401063300103

9. The Microalbuminuria Collaborative Study Group (1999) Predictors of the development of microalbuminuria in patients with Type 1 diabetes mellitus: a seven-year prospective study. Diabetic Med J British Diabetic Assoc 16(11):918-925

10. Rossing P, Hougaard P, Parving HH (2002) Risk factors for development of incipient and overt diabetic nephropathy in type 1 diabetic patients: a 10-year prospective observational study. Diabetes Care 25(5):859-864. https://doi.org/10.2337/diacare.25.5.859

11. Giorgino F, Laviola L, Cavallo Perin P, Solnica B, Fuller J, Chaturvedi N (2004) Factors associated with progression to macroalbuminuria in microalbuminuric Type 1 diabetic patients: the EURODIAB prospective complications study. Diabetologia 47(6):1020-1028. https://doi.org/10.1007/s00125-004-1413-8

12. Nordwall M, Arnqvist HJ, Bojestig M, Ludvigsson J (2009) Good glycemic control remains crucial in prevention of late diabetic complications-the Linköping diabetes complications study. Pediatr Diabetes 10(3):168-176. https://doi.org/10.1111/j.1399-5448. 2008.00472.x

13. Thomas MC, Rosengård-Bärlund M, Mills V (2006) Serum lipids and the progression of nephropathy in type 1 diabetes. Diabetes 
Care 29(2):317-322. https://doi.org/10.2337/diacare.29.02.06. dc05-0809

14. Skrivarhaug T, Bangstad HJ, Stene LC, Sandvik L, Hanssen KF, Joner G (2006) Low risk of overt nephropathy after 24 yr of childhood-onset type 1 diabetes mellitus (T1DM) in Norway. Pediatr Diabetes 7(5):239-246. https://doi.org/10.1111/j.1399-5448.2006. 00204.x

15. Raile K, Galler A, Hofer S et al (2007) Diabetic nephropathy in 27,805 children, adolescents, and adults with type 1 diabetes: effect of diabetes duration, A1C, hypertension, dyslipidemia, diabetes onset, and sex. Diabetes Care 30(10):2523-2528. https://doi. org/10.2337/dc07-0282

16. Perkins BA, Bebu I, de Boer IH et al (2019) Risk factors for kidney disease in type 1 diabetes. Diabetes Care 42(5):883-890. https://doi.org/10.2337/dc18-2062

17. Unger T, Borghi $C$, Charchar F et al (2020) 2020 International society of hypertension global hypertension practice guidelines. Hypertension 75(6):1334-1357

18. Williams B, Mancia G, Spiering W et al (2018) 2018 ESC/ESH Guidelines for the management of arterial hypertension. Eur Heart J 39(33):3021-3104. https://doi.org/10.1093/eurheartj/ehy339

19. American Diabetes Association (2021) 10 Cardiovascular disease and risk management: standards of medical care in diabetes-2021. Diabetes Care 44(Suppl 1):S125-S150. https://doi.org/10.2337/ dc21-S010

20. Lind M, Pivodic A, Svensson AM, Ólafsdóttir AF, Wedel H, Ludvigsson J (2019) HbA1c level as a risk factor for retinopathy and nephropathy in children and adults with type 1 diabetes: Swedish population based cohort study. BMJ (Clin Res ed) 366:14894. https://doi.org/10.1136/bmj.14894

21. Samuelsson U, Åkesson K, Peterson A, Hanas R, Hanberger L (2018) Continued improvement of metabolic control in Swedish pediatric diabetes care. Pediatr Diabetes 19(1):150-157. https:// doi.org/10.1111/pedi.12467

22. Eeg-Olofsson K, Cederholm J, Nilsson PM et al (2010) Glycemic control and cardiovascular disease in 7454 patients with type 1 diabetes: an observational study from the Swedish National Diabetes Register (NDR). Diabetes Care 33(7):1640-1646. https:// doi.org/10.2337/dc10-0398

23. Jeppsson JO, Kobold U, Barr J et al (2002) Approved IFCC reference method for the measurement of $\mathrm{HbA1c}$ in human blood. Clin Chem Laborat Med 40(1):78-89. https://doi.org/10.1515/CCLM. 2002.016

24. Lindblad B, Nordin G (2013) External quality assessment of $\mathrm{HbA1c}$ and its effect on comparison between Swedish pediatric diabetes clinics. Experiences from the Swedish pediatric diabetes quality register (Swediabkids) and Equalis. Clin Chem Laborat Med 51(10):2045-2052. https://doi.org/10.1515/cclm-2013-0226

25. Hopper JL (2015) Odds per adjusted standard deviation: comparing strengths of associations for risk factors measured on different scales and across diseases and populations. Am J Epidemiol 182(10):863-867. https://doi.org/10.1093/aje/kwv193
26. Guy J, Ogden L, Wadwa RP et al (2009) Lipid and lipoprotein profiles in youth with and without type 1 diabetes: the SEARCH for Diabetes in Youth case-control study. Diabetes Care 32(3):416420. https://doi.org/10.2337/dc08-1775

27. Mäkinen VP, Soininen P, Kangas AJ et al (2013) Triglyceride-cholesterol imbalance across lipoprotein subclasses predicts diabetic kidney disease and mortality in type 1 diabetes: the FinnDiane Study. J Inter Med 273(4):383-395. https://doi.org/10.1111/joim. 12026

28. Vergès B (2020) Dyslipidemia in type 1 diabetes: AMaskedDanger. Trends Endocrinol Metab 31(6):422-434. https://doi.org/10. 1016/j.tem.2020.01.015

29. Hartz JC, de Ferranti S, Gidding S (2018) Hypertriglyceridemia in diabetes mellitus: implications for pediatric care. J Endocrine Soc 2(6):497-512. https://doi.org/10.1210/js.2018-00079

30. Rosina R, Crisp J, Steinbeck K (2003) Treatment adherence of youth and young adults with and without a chronic illness. Nurs Health Sci 5(2):139-147. https://doi.org/10.1046/j.1442-2018. 2003.00149.x

31. Grassi G, Mancia G, Nilsson PM (2016) Specific blood pressure targets for patients with diabetic nephropathy? Diabetes Care 39(Suppl 2):S228-S233. https://doi.org/10.2337/dcS15-3020

32. Wiegman A, Gidding SS, Watts GF et al (2015) Familial hypercholesterolaemia in children and adolescents: gaining decades of life by optimizing detection and treatment. Eur Heart J 36(36):2425-2437. https://doi.org/10.1093/eurheartj/ehv157

33. Vestberg D, Rosengren A, Eeg-Olofsson K et al (2018) Body mass index as a risk factor for coronary events and mortality in patients with type 1 diabetes. Open Heart 5(1):e000727. https://doi.org/10. 1136/openhrt-2017-000727

34. Rosengren A, Vestberg D, Svensson AM et al (2015) Longterm excess risk of heart failure in people with type 1 diabetes: a prospective case-control study. Lancet Diabetes Endocrinol 3(11):876-885. https://doi.org/10.1016/S2213-8587(15)00292-2

35. Vestberg D, Rosengren A, Olsson M, Gudbjörnsdottir S, Svensson AM, Lind M (2013) Relationship between overweight and obesity with hospitalization for heart failure in 20,985 patients with type 1 diabetes: a population-based study from the Swedish National Diabetes Registry. Diabetes Care 36(9):2857-2861. https://doi. org/10.2337/dc12-2007

36. Hill CJ, Cardwell CR, Maxwell AP et al (2013) Obesity and kidney disease in type 1 and 2 diabetes: an analysis of the National Diabetes Audit. QJM Monthly J Assoc Phys 106(10):933-942. https://doi.org/10.1093/qjmed/hct123

37. Harsha DW, Bray GA (2008) Weight loss and blood pressure control (Pro). Hypertension 51(6):1420-1425. https://doi.org/10. 1161/HYPERTENSIONAHA.107.094011

Publisher's Note Springer Nature remains neutral with regard to jurisdictional claims in published maps and institutional affiliations.

\section{Authors and Affiliations}

\section{Shilan Seyed Ahmadi 1,2,11 (1) $\cdot$ Aldina Pivodic ${ }^{3,4} \cdot$ Ann-Marie Svensson ${ }^{5} \cdot$ Hans Wedel $^{6} \cdot$ Björn Rathsman $^{7}$. Thomas Nyström ${ }^{8}$. Johnny Ludvigsson ${ }^{9} \cdot$ Marcus Lind $^{1,2,10}$}

1 Department of Molecular and Clinical Medicine, Institute of Medicine, University of Gothenburg, Gothenburg, Sweden
2 Department of Internal Medicine, Sahlgrenska University Hospital, Gothenburg, Sweden

3 Statistiska Konsultgruppen, Gothenburg, Sweden 
4 Department of Clinical Neuroscience, Institute of Neuroscience and Physiology, Sahlgrenska Academy, University of Gothenburg, Gothenburg, Sweden

5 Centre of Registers in Region Västra Götaland, Gothenburg, Sweden

6 Department of Health Metrics, Sahlgrenska Academy, University of Gothenburg, Gothenburg, Sweden

7 Department of Clinical Science and Education, Sachs' Children and Youth Hospital, Södersjukhuset, Karolinska Institutet, Stockholm, Sweden
8 Department of Clinical Science and Education, Internal Medicine, Södersjukhuset, Karolinska Institutet, Stockholm, Sweden

9 Department of Biomedical and Clinical Sciences, Crown Princess Victoria Children's Hospital, and Division of Paediatrics, Linköping University, Linköping, Sweden

10 Department of Medicine, NU Hospital Group, Uddevalla, Sweden

11 Department of Medicine, Uddevalla Hospital, 45180 Uddevalla, Sweden 